\title{
Erratum to: Sub-classification of Stage II colorectal cancer based on clinicopathological risk factors for recurrence
}

Takuzo Hashimoto • Michio Itabashi •

Shinpei Ogawa - Tomoichiro Hirosawa •

Yoshiko Bamba · Satoru Shimizu - Shingo Kameoka

Published online: 20 June 2014

(C) Springer Japan 2014

Erratum to: Surg Today

DOI 10.1007/s00595-013-0807-y

The original version of this article unfortunately contained a mistake.

Dr. Michio Itabashi should be indicated as the corresponding author.

The online version of the original article can be found under doi:10.1007/s00595-013-0807-y.

T. Hashimoto $\cdot$ M. Itabashi $(\bowtie) \cdot$ S. Ogawa $\cdot$ T. Hirosawa .

Y. Bamba · S. Kameoka

Department of Surgery II, Tokyo Women's Medical University,

8-1 Kawadacho, Shinjuku-ku, Tokyo 162-8666, Japan

e-mail: itabashi@surg2.twmu.ac.jp

T. Hashimoto

e-mail: hashizou@m3.dion.ne.jp; hashizou@surg2.twmu.ac.jp

S. Shimizu

Tokyo Women's Medical University Medical Research Institute,

Tokyo, Japan 